\title{
Noninvasive Ventilation Use Among Medicare Beneficiaries at the End of Life
}

\author{
Sullivan DR, Kim H, Gozalo $P$, et al. Trends in noninvasive and invasive mechanical ventilation \\ among Medicare beneficiaries at the end of life. JAMA Intern Med. 2021;181:93-102. \\ doi:10.1001/jamainternmed.2020.5640
}

\section{Study Overview}

Objective. To examine the trend of noninvasive and invasive mechanical ventilation at the end of life from 2000 to 2017.

Design. Observational population-based cohort study.

Setting and participants. The study was a population-based cohort study to examine the use of noninvasive and invasive mechanical ventilation among decedents. The study included a random 20\% sample of Medicare beneficiaries older than 65 years who were hospitalized in the last 30 days of life and died between January 1, 2000, and December 31, 2017, except for the period October 1, 2015, to December 31, 2015, when the transition from International Classification of Diseases, Ninth Revision (ICD-9) to ICD-10 occurred. Beneficiaries with the primary admitting diagnosis of cardiac arrest or with preexisting tracheostomy were excluded because of expected requirements for ventilatory support. The sample included a total of 2,470,735 Medicare beneficiaries; mean age was 82.2 years, and $54.8 \%$ were female. Primary admitting diagnosis codes were used to identify 3 subcohorts: congestive heart failure, chronic obstructive pulmonary disease, and cancer; a fourth subcohort of dementia was identified using the primary admitting diagnosis code or the first 9 secondary diagnosis codes.
Main outcome measures. The study used procedure codes to identify the use of noninvasive ventilation, invasive mechanical ventilation, or none among decedents who were hospitalized in the last 30 days of life. Descriptive statistics to characterize variables by year of hospitalization and ventilatory support were calculated, and the rates of noninvasive and invasive mechanical ventilation use were tabulated. Other outcomes of interest include site of death (in-hospital death), hospice enrollment at death, and hospice enrollment in the last 3 days of life as measures of end-of- life care use. Multivariable logistic regressions were used to examine noninvasive and invasive mechanical ventilation use among decedents, and time trends were examined, with the pattern of use in year 2000 as reference. Subgroup analysis with the subcohort of patients with different diagnoses were conducted to examine trends.

Main results. From 2000 to 2017, 16.3\% of decedents had invasive mechanical ventilation, 3.7\% had noninvasive ventilation, and $1.0 \%$ had both noninvasive and invasive ventilation during their hospital stay. Compared to the reference year 2000, there was a 9-fold increase in noninvasive ventilation use, from $0.8 \%$ to $7.1 \%$ in 2017 , and invasive mechanical ventilation use also increased slightly, from $15.0 \%$ to 18.5\%. Compared to year 2000, decedents were 2.63

\section{Outcomes Research in Review SECTION EDITORS}

KatRina F. MATEO, PHD, MPH

CUNY School of Public Health New York, NY

TAISHI HIRAI, MD

University of Missouri

Columbia, MO
DANIEL ISAAC, DO, MS

Michigan State University East Lansing, Ml

WiLLIAM HUNG, MD, MPH

Icahn School of Medicine at Mount Sinai New York, NY
FRED Ko, MD, MS

Icahn School of Medicine at Mount Sinai New York, NY

GORDON NGAI, MD, MPH

Icahn School of Medicine at Mount Sinai New York, NY 


\section{Outcomes Research in Review}

times and 1.04 times (adjusted odds ratio [OR]) more likely to receive noninvasive ventilation and invasive mechanical ventilation, respectively, in 2005, 7.87 times and 1.39 times more likely in 2011, and 11.84 times and 1.63 times more likely in 2017.

Subgroup analysis showed that for congestive heart failure and chronic obstructive pulmonary disease, the increase in noninvasive ventilation use mirrored the trend observed for the overall population, but the use of invasive mechanical ventilation did not increase from 2000 to 2017 , with a rate of use of $11.1 \%$ versus $7.8 \%$ (adjusted OR, 1.07; 95\% confidence interval [Cl], 0.95-1.19) for congestive heart failure and $17.4 \%$ vs $13.2 \%$ (OR 1.03, 95\% Cl, 0.88-1.21) for chronic obstructive pulmonary disease. For the cancer and dementia subgroups, the increase in noninvasive ventilation use from 2000 to 2017 was accompanied by an increase in the use of invasive mechanical ventilation, with a rate of $6.2 \%$ versus $7.4 \%$ (OR, 1.40; 95\% Cl, 1.26-1.55) for decedents with cancer and a rate of $5.7 \%$ versus $6.2 \%(\mathrm{OR}, 1.28 ; 95 \% \mathrm{Cl}, 1.17-$ 1.41) for decedents with dementia. For other measures of end-of-life care, noninvasive ventilation use when compared to invasive mechanical ventilation use was associated with lower rates of in-hospital (acute care) deaths (50.3\% vs $76.7 \%)$, hospice enrollment in the last 3 days of life (late hospice enrollment; $57.7 \%$ vs $63.0 \%$ ), and higher rates of hospice enrollment at death (41.3\% vs $20.0 \%)$.

Conclusion. There was an increase in the use of noninvasive ventilation from 2000 through 2017 among Medicare beneficiaries who died. The findings also suggest that the use of invasive mechanical ventilation did not increase among decedents with congestive heart failure and chronic obstructive pulmonary disease but increased among decedents with cancer and dementia.

\section{Commentary}

Noninvasive ventilation offers an alternative to invasive mechanical ventilation for providing ventilatory support for respiratory failure, and may offer benefits as it could avert adverse effects associated with invasive mechanical ventilation, particularly in the management of respiratory failure due to congestive heart failure and chronic obstructive pulmonary disease. ${ }^{1}$ There is evidence for potential benefits of use of noninvasive ventilation in other clinical scenarios, such as pneumonia in older adults with comorbidities, though its clinical utility is not as well established for other diseases. ${ }^{2}$

As noninvasive ventilation is introduced into clinical practice, it is not surprising that over the period of the study (2000 to 2017) that its use increased substantially. Advance directives that involve discussion of life-sustaining treatments, including in scenarios with respiratory failure, may also result in physician orders that specify whether an individual desires invasive mechanical ventilation versus other medical treatments, including noninvasive ventilation. ${ }^{3,4}$ By examining the temporal trends of use of noninvasive and invasive ventilation, this study reveals that invasive mechanical ventilation use among decedents with dementia and cancer has increased, despite increases in the use of noninvasive ventilation. It is important to understand further what would explain these temporal trends and whether the use of noninvasive and also invasive mechanical ventilation at the end of life represents appropriate care with clear goals or whether it may represent overuse. It is also less clear in the end-of-life care scenario what the goals of treatment with noninvasive ventilation would be, especially if it does not avert the use of invasive mechanical ventilation.

The study includes decedents only, thus limiting the ability to draw conclusions about clinically appropriate care. ${ }^{5}$ Further studies should examine a cohort of patients who have serious and life-threatening illness to examine the trends and potential effects of noninvasive ventilation on outcomes and utilization, as individuals who have improved and survived would not be included in this present decedent cohort.

\section{Applications for Clinical Practice}

This study highlights changes in the use of noninvasive and invasive ventilation over time and the different trends seen among subgroups with different diagnoses. For older adults with serious comorbid illness such as dementia, it is especially important to have discussions on advance directives so that care at the end of life is concordant with the patient's wishes and that unnecessary, burdensome care can be averted. Further studies to understand and define the appropriate use of noninvasive and invasive mechanical ventilation for older adults with significant comorbidi- 
ties who have serious, life-threatening illness are needed to ensure appropriate clinical treatment at the end of life. -William W. Hung, MD, MPH

doi:10.12788/jcom.0037

\section{References}

1. Lindenauer PK, Stefan MS, Shieh M et al. Outcomes associated with invasive and noninvasive ventilation among patients hospitalized with exacerbations of chronic obstructive pulmonary disease. JAMA Intern Med. 2014;174:1982-993.
2. Johnson CS, Frei CR, Metersky ML, et al. Non-invasive mechanical ventilation and mortality in elderly immunocompromised patients hospitalized with pneumonia: a retrospective cohort study. BMC Pulm Med. 2014;14:7. Published 2014 Jan 27. doi:10.1186/1471-2466-14-7

3. Lee R, Brumbeck L, Sathitratanacheewin S, et al. Association of physician orders for life-sustaining treatment with icu admission among patients hospitalized near the end of life. JAMA. 2020;323:950-60.

4. Bomba P, Kemp M, Black J. POLST: An improvement over traditional advance directives. Cleveland Clinic J Med. 2012;79:457-464.

5. Duncan I, Ahmed T, Dove H, Maxwell TL. Medicare cost at end of life. Am J Hosp Palliat Care. 2019;36:705-710.

\section{Theory of Planned Behavior Provides A Theoretical Explanation For Enhanced Behavior Change With Genetic-Based Lifestyle Interventions}

Horne JR, Gilliland JA, Vohl MC, Madill J. Exploring attitudes, subjective norms and perceived behavioural control in a genetic-based and a population-based weight management intervention: a one-year randomized controlled trial. Nutrients. 2020;12:3768.

\section{Study Overview}

objective. To determine the impact of providing genetically tailored and population-based lifestyle advice for weight management on key constructs of the Theory of Planned Behavior (TPB), a widely accepted theory used to help predict human lifestyle-related behaviors.

Design. Pragmatic, cluster, randomized controlled trial.

Settings and participants. This study took place at the East Elgin Family Health Team, a primary care clinic in Aylmer, Ontario, Canada. Recruitment occurred between April 2017 and September 2018, with staggered intervention cohorts occurring from May 2017 to September 2019. Participants enrolled in a weight management program at the clinic were invited to participate in the study if they met the following inclusion criteria: body mass index (BMI) $\geq 25 \mathrm{~kg} / \mathrm{m}^{2},>18$ years of age, English-speaking, willing to undergo genetic testing, having access to a computer with internet at least 1 day per week, and not seeing another health care provider for weight loss advice outside of the study. Exclusion criteria included pregnancy and lactation. All participants provided written informed consent.

Interventions. At baseline, weight management program cohorts (average cohort size was 14 participants) were randomized (1:1) to receive either the standard population-based intervention (Group Lifestyle Balance, or GLB) or a modified GLB intervention, which included the provision of lifestyle genomics ( $L G X)$ information and advice (GLB+LGX). Both interventions aimed to assist participants with weight management and healthy lifestyle change, with particular focus on nutrition and physical activity (PA). Interventions were 12 months long, consisting of 23 group-based sessions and 3 one-on-one sessions with a registered dietitian after 3 , 6, and 12 months (all sessions were face-to-face). To improve intervention adherence, participants were given reminder calls for their one-on-one appointments and for the start of their program. A sample size was calculated based on the primary outcome indicating that a 


\section{Outcomes Research in Review}

total of 74 participants were needed ( $n=37$ per group) for this trial. By September 2019, this sample size was exceeded with 10 randomized groups $(n=140)$.

The 5 randomized standard GLB groups followed the established GLB program curriculum comprising population-based information and advice while focusing on following a calorie-controlled, moderate-fat (25\% of calories) nutrition plan with at least 150 minutes of weekly moderate-intensity PA. Participants were also provided with a 1-page summary report of their nutrition and PA guidelines at the first group meeting outlining population-based targets, including acceptable macronutrient distribution ranges for protein, total fat, saturated fat, monounsaturated fat, polyunsaturated fat, sodium, calories, snacking, and PA.

The 5 randomized modified GLB+LGx groups followed a modified GLB program curriculum in which participants were given genetic-based information and advice, which differed from the advice given to the standard GLB group, while focusing on following a calorie-controlled nutrition plan. The nutrition and PA targets were personalized based on their individual genetic variation. For example, participants with the AA variant of FTO (rs9939609) were advised to engage in at least 30 to 60 minutes of PA daily 6 days per week, with muscle-strengthening activities at least 2 days per week, rather than receiving the standard population-based advice to aim for 150 minutes weekly of PA with at least 2 days per week of muscle-strengthening activity. Participants were also provided with a 1-page summary report of their nutrition and PA guidelines at the first group meeting, which outlined genetic-based information and advice related to protein, total fat, saturated fat, monounsaturated fat, polyunsaturated fat, sodium, calories, snacking, and PA.

Measures and analysis. Change in the TPB components (attitudes, subjective norms and perceived behavioral control) were measured via a TPB questionnaire at 5 time points: baseline (2-week run-in period), immediately after the first group session (where participants received a summary report of either population-based or genetic-based recommendations depending on group assignment), and after 3-, 6- and 12-month follow-ups.
Attitudes, subjective norms, and perceived behavioral control were measured on a Likert scale from 1 through 7. Self-reported measures of actual behavioral control (including annual household income, perceptions about events arising in one's day-to-day life that suddenly take up one's free time, perceptions about the frequency of feeling ill or tired, and highest achieved level of education) were collected via survey questions and assessed on a Likert scale of 1 through 7. Stage of change was also measured, based on the Transtheoretical Model, using a Likert scale of 1 through 6.

Linear mixed models were used to conduct withinand between-group analyses using SPSS version 26.0, while controlling for measures of actual behavioral control. All analyses were intention-to-treat by originally assigned groups, with mean value imputation conducted for missing data. A Bonferroni correction for multiple testing was used. For all statistical analyses, the level of significance was set at $P<0.05$ and trending towards significance at $P=0.05-0.06$.

Main results. Participants consisted of primarily middle-age, middle-income, Caucasian females. Baseline attitudes towards the effectiveness of nutrition and PA for weight management were generally positive, and participants perceived that undergoing genetic testing would assist with weight management. Participants had overall neutral subjective norms related to friends and family consuming a healthy diet and engaging in PA, but perceived that their friends, family, and health care team (HCT) believed it was important for them to achieve their nutrition and PA recommendations. Participants overall also perceived that their HCT believed genetic testing could assist with weight management. Baseline measures of perceived behavioral control were overall neutral, with baseline stage of change between "motivation" and "action" (short-term; <3 months).

In within-group analyses, significant improvements $(P<0.05)$ in attitudes towards the effectiveness of nutrition and PA recommendations for weight management, subjective norms related to both friends and family consuming a healthy diet, and perceived behavioral control in changing PA/dietary intake and managing weight tended to be short-term in the GLB group and long- 


\section{Outcomes Research in Review}

term for the GLB+LGx group. In all cases of betweengroup differences for changes in TPB components, the GLB group exhibited reductions in scores, whereas the GLB+LGx group exhibited increases or improvements. Between-group differences (short-term and long-term) in several measures of subjective norms were observed. For example, after 3 months, significant between-group differences were observed in changes in perception that friends believed LGx would help with weight management $(P=0.024)$. After 12 months, between-group differences trending towards significance were also observed in changes in perception that family members believed genetic testing would help with weight management $(P=0.05)$. Significant between-group differences and differences trending towards significance were also observed at 12 months for changes in perception that family believed it was important for the participant to achieve the PA recommendations $(P=0.049)$ and nutrition recommendations $(P=0.05)$. Betweengroup differences trending towards significance were also observed at 3 months in attitudes towards the effectiveness of LGx for weight management $(P=0.06)$. There were no significant between-group differences observed in changes in perceived behavioral control.

Conclusion. Results from this study support the hypothesis that the TPB can help provide a theoretical explanation for why genetically tailored lifestyle information and advice can lead to improvements in lifestyle behavior change.

\section{Commentary}

Because health behaviors are critical in areas such as prevention, treatment, and rehabilitation, it is important to describe and understand what drives these behaviors. ${ }^{1}$ Theories are important tools in this effort as they aim to explain and predict health behavior and are used in the design and evaluation of interventions. ${ }^{1}$ The TPB is one of the most widely accepted behavior change theories and posits that attitudes, subjective norms (or social pressures and behaviors), and perceived behavioral control are significant predictors of an individual's intention to engage in behaviors. ${ }^{2}$ TPB has been highlighted in the literature as a validated theory for predicting nutrition and PA intentions and resulting behaviors..$^{3,4}$
Motivating lifestyle behavior change in clinical practice can be challenging, but some studies have demonstrated how providing genetic information and advice (or lifestyle genomics) can help motivate changes in nutrition and PA among patients. ${ }^{5-7}$ Because this has yet to be explained using the TPB, this study is an important contribution to the literature as it aimed to determine the impact of providing genetically tailored and population-based lifestyle advice for weight management on key constructs of the TPB. Briefly, results from within-group analyses in this study demonstrated that the provision of genetically tailored lifestyle information and advice (via the GLB+LGx intervention) tended to impact antecedents of behavior change, more so over the long-term, while population-based advice (via the standard GLB intervention) tended to impact antecedents of behavior change over the short-term (eg, attitudes towards dietary fat intake, perceptions that friends and family consume a healthy diet, and perceptions about the impact of genetic-based advice for weight management). In addition, between-group differences in subjective norms observed at 12 months suggested that social pressures and norms may be influencing long-term changes in lifestyle habits.

While key strengths of this study include its pragmatic cluster randomized controlled trial design, 12-month intervention duration, and intent-to-treat analyses, there are some study limitations, which are acknowledged by the authors. Generalizability is limited to the demographic characteristics of the study population (ie, middle-aged, middle-income, Caucasian females enrolled in a lifestyle change weight management program). Thus, replication of the study is needed in more diverse study populations and with health-related outcomes beyond weight management. In addition, as the authors indicate, future research should ensure the inclusion of theory-based questionnaires in genetic-based intervention studies assessing lifestyle behavior change to elucidate theory-based mechanisms of change.

\section{Applications for Clinical Practice}

Population-based research has consistently indicated that nutrition interventions typically impact short-term 
dietary changes. Confronting the challenge of longterm adherence to nutrition and PA recommendations requires an understanding of factors impacting longterm motivation and behavior change. With increased attention on and research into genetically tailored lifestyle advice (or lifestyle genomics), it is important for clinical practitioners to be familiar with the evidence supporting these approaches. In addition, this research highlights the need to consider individual factors (attitudes, subjective norms, and perceived behavioral control) that may predict successful change in lifestyle habits when providing nutrition and PA recommendations, whether population-based or genetically tailored.

Katrina F. Mateo, $P h D, M P H$

doi:10.12788/jcom.0038

\section{References}

1. Lippke S, Ziegelmann JP. Theory-based health behavior change: Developing, testing, and applying theories for evidence-based interventions. Appl Psychol. 2008;57:698-716.

2. Ajzen I. The Theory of planned behaviour: reactions and reflections. Psychol Health. 2011;26:1113-1127.

3. McDermott MS, Oliver M, Simnadis T, et al. The Theory of Planned Behaviour and dietary patterns: A systematic review and meta-analysis. Prev Med (Baltim). 2015;81:150-156.

4. McEachan RRC, Conner M, Taylor NJ, Lawton RJ. Prospective prediction of health-related behaviours with the theory of planned behaviour: A meta-analysis. Health Psychol Rev. 2011;5:97-144.

5. Hietaranta-Luoma H-L, Tahvonen R, Iso-Touru T, et al A. An intervention study of individual, APOE genotype-based dietary and physical-activity advice: impact on health behavior. J Nutrigenet Nutrigenomics. 2014;7:161-174.

6. Nielsen DE, El-Sohemy A. Disclosure of genetic information and change in dietary intake: a randomized controlled trial. DeAngelis MM, ed. PLoS One. 2014;9(11):e112665.

7. Egglestone C, Morris A, O'Brien A. Effect of direct-to-consumer genetic tests on health behaviour and anxiety: a survey of consumers and potential consumers. J Genet Couns. 2013;22:565-575.

\section{CALL for REVIEWERS}

The Journal of Clinical Outcomes Management welcomes applications

from physicians, directors of pharmacy, nurse leaders,

and others working in ambulatory, inpatient, and long-term care

to serve as peer reviewers.

\section{You will not be asked to review more than 3 times per year.}

The following medical specialties are especially of interest: cardiology, critical care, endocrinology, hematology/oncology, hospital medicine, infectious diseases, mental health, neurology, nephrology, pulmonary disease, and rheumatology.

To apply to be a peer reviewer, email your $\mathrm{CV}$ along with a description of your subject areas of interest to rlitchkofski@mdedge.com.

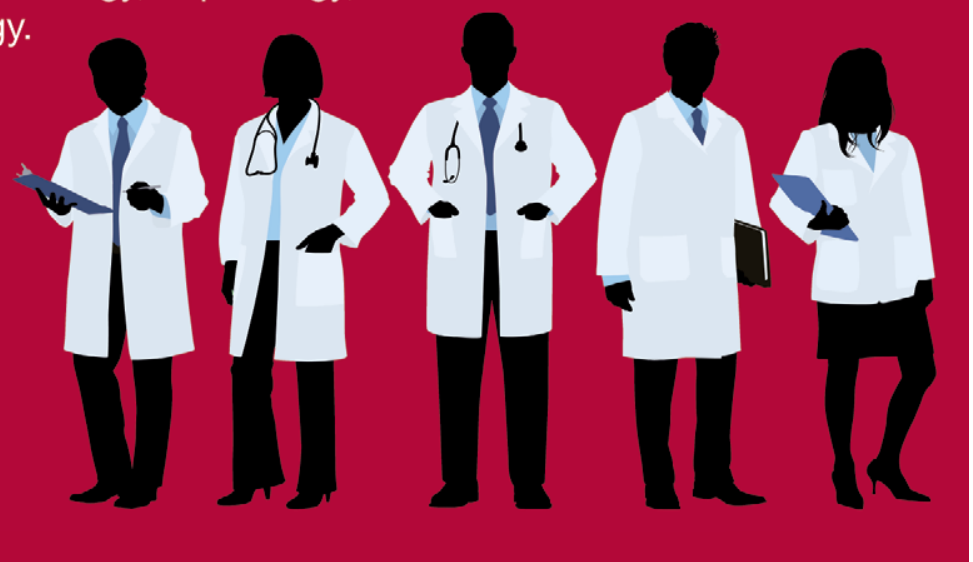

